\title{
Zeit meines Lebens - Das Gebet des Herzens als Ort der Entstehung von Zeit
}

\author{
Eine Fingerübung ${ }^{1}$
}

Johanna Breidenbach

\section{Die Fragestellung}

Das Gebet ist nach Martin Luther das Herz des christlichen Glaubens. Im Folgenden sollen diese drei Begriffe - Gebet, Herz, Glaube unter dem Aspekt der Zeitlichkeit entfaltet werden. Diese kommt durch die berühmt gewordene Ermunterung in 1 Thess 5,17 ohne Unterlass zu beten ins Spiel, einem der wirkmächtigsten Verse in der Gebetsliteratur.

Der Stein des Anstosses furr diese Frage ist die Wahrnehmung zweier schwer miteinander zu vermittelnden Glaubens- und Gebetsverständnisse, die holzschnittartig Martin Luther und Friedrich Schleiermacher zugeordnet werden. Das eine lässt Glaube und Gebet ganz auf dem Wort aufruhen, das so kräftig und lebendig es auch sein mag, wie kaum etwas anderes der Vergänglichkeit ausgeliefert ist: kaum ist es erklungen, so ist auch schon wieder weg. Das ruft nach ständiger Erneuerung und unermüdlicher Wiederholung. Das andere lässt Gebet ganz in das Bewusstsein aufgehen und zieht es ab von seiner äusserlichen sinnlichen Anstössigkeit. Das Gebet wird zur einer Domäne der Innerlichkeit und damit der Zeitlichkeit weitestgehend enthoben. Unablässigkeit wird zu einer Funktion des Bewusstseins.

In der Tradition des Herzensgebets geht es um eine Verbindung von Wort und Herz in einer spezifischen Praxis leiblicher Vermittlung, und die These der folgenden Überlegungen ist, dass sich mit dem Herzensgebet eine mögliche Versöhnung der beiden soeben genannten Positionen andeuten lässt.

Im Verlauf der Abschnitte wird deutlich werden, wie vielfältig die Bezüge zwischen Herz, Glaube, Wort und Zeit sind und sich kaum

\footnotetext{
1 Ich danke Andreas Hunziker für wichtige Impulse und Hinweise im Gespräch über das Gebet.
} 
in das hier angelegte enge Schema einpassen lassen wollen, ohne dass ihnen jedoch gebührend nachgegangen werden kann.

\section{Unablässigkeit durch Rhythmisierung: Glaube und Gebet bei Martin Luther}

\section{a. Wort und Glaube}

Der wesentliche Zusammenhang von Wort und Glaube bei Luther sind der Leserin, so wird hier vorausgesetzt, hinlänglich bekannt. Die folgenden Ausführungen verstehen sich also nur als andeutende Erinnerungshilfe und lehnen sich dabei an die Ausführungen von Joachim Ringleben an. ${ }^{2}$ Dabei werde ich insbesondere auf den immer wieder hervortretenden Aspekt der Zeitlichkeit achten. Es wird dabei deutlich werden, wie durch die sinnliche Gestalt des Glaubens die Zeit eine bedeutende Rolle für das Gebet gewinnt insofern beide angewiesen sind auf das Vorhandensein mündlich oder schriftlich zukommender Worte, so dass es anhebt und wieder aufhört und damit Unablässigkeit durch Rhythmus gewinnt.

Wort und Glaube gehören bei Luther zutiefst zusammen. Es geht dabei auch um die Glaubensinhalte, die von den Worten vermittelt werden, vor allem aber um die Wortförmigkeit des Glaubens selbst. Sie drückt sich aus in der wesentlichen Bezogenheit auf das Evangelium, durch welches der Geist den Menschen zum Glauben beruft. ${ }^{3}$ Dadurch ist dem Wort nicht nur seine inhaltliche Bestimmung verliehen - die promissiones der Vergebung der Sünden und Versöhnung mit Gott durch Kreuz und Auferstehung Jesu Christi -, sondern auch die formale Prägung des Glaubens als exzentrische: "nicht aus eigener Vernunft und Kraft" gelangt man zum Hören auf das Wort. Mit dem extra me des Wortes ist aber notwendig verbunden auch das pro me. Nur insofern es sich mir als Anrede (einer von mir differenten Stimme) zu verstehen gibt, ${ }^{4}$ kann es auch die Gewissheit

\footnotetext{
2 Joachim Ringleben, Gott im Wort. Luthers Theologie von der Sprache her, Tübingen 2010, Kapitel X, Wort und Glaube, 444-484.

3 Martin Luther, Der Kleine Katechismus, BSLK 511, 46-512,3: „Ich glaube, dass ich nicht aus eigener Vernunft noch Kraft an Jesum Christ, meinen Herrn, glauben oder zu ihm kommen kann, sondern der heilige Geist hat mich durchs Evangelium berufen $[\ldots]$.."

4 Bei einer vorhergehenden Erörterung des Anredegeschehens des Wortes (beim Lesen) macht Ringlebens Wortwahl (Geschehen, Ereignis) auf die spezifische Zeit-
} 
schaffen, dass gerade ich von jenem Wort der Versöhnung gemeint bin. Eben jenes persönliche Betroffen- bzw. Ergriffensein vom Wort meint Luther mit der Rede vom `Herz . Das Herz ist der Ort, an dem die Worte geistlich verköstigt werden ${ }^{5}$ und dem Vertrauen zu Wachstum verhelfen, mit dem sich der Mensch in allen Nöten an Gott wenden und auf ihn verlassen mag. ${ }^{6}$ Dabei gilt, wie unten bei der Dialektik von Zeitgewährung und Zeitentzug noch einmal zu sehen sein wird, dass das Herz die Mitte ${ }^{7}$ des Menschen meint, die im Glauben gerade durch ihre Verschiebung (auf Gottes Wort hin, das dem Herz von aussen her zukommt) ihren Ort findet. ${ }^{8}$ Dem entspricht der Respons des Glaubensbekenntnisses in der 1. Pers. Sg. Es ist also das Wort, auf welches sich im Glauben zu hören komme, in dem Gott Gemeinschaft mit mir hat und die sich eben wegen ihrer sprachlichen Begründung und Verfassung als "Gemeinschaft in Kommunikation " ${ }^{9}$ entfaltet. Wichtig daran ist nun vor allem Ringlebens Weiterführung, dass das Gespräch das Sein Jesu Christi thematisiert und damit Gottes Sein ins Werden verschiebt: „Das >verbum ‘ vom Anfang ist mithin ein >Tätigkeitswort` und ein zeitliches Wort. Gott und sein ewiges Wort übersetzen sich ins Zeit-Wort. «10 Zeit kommt hier also in den Blick als geschöpfliche Bedingung des Gesprächs, welche in den Grenzen menschlichen Kommunikationsvermögens begründet liegt: das Gespräch wird begonnen, kann sich vertiefen und entfalten, wird unterbrochen (wenn es nicht gar abgebrochen wird) und wird neu angeknüpft. So entspricht es dem

förmigkeit dieses Vorgangs aufmerksam. Ringleben, Gott im Wort, $227 \mathrm{f}$.

5 Martin Luther, Dass diese Worte Christi "Das ist mein Leib « noch fest stehen [1527], WA 23, 179, 27-29: »Nu die wort kann freylich niemand durch den hals ynn den bauch jagen, sondern mus sie durch die oren ins hertz fassen. Was fasset er aber yns hertz durch die wort? Nichts anders denn das sie lauten, nemlich sden leib fur uns gegeben«, welchs ist das geistliche essen." Ringleben, Gott im Wort, 455f, mit weiteren Verweisen.

6 Martin Luther, Der Grosse Katechismus, BSLK 560, 10-13: "Ein Gott heißet das, dazu man sich versehen soll alles Guten und Zuflucht haben in allen Nöten.»

7 Ringleben, Gott im Wort, 454: „Mitte seines Personseins«; 483: »Mitte seiner Affektivität«.

${ }^{8}$ Martin Luther, Resolutio disputationis de fide infusa et acquisita [1520], WA 6,94,10f: NVerum quando verbum Dei sonat, quod veritas est, et cor ei adhaeret per fidem, tunc cor imbuitur eadem veritate verbi«.

9 Ringleben, Gott im Wort, 450, mit Bezug auf Luthers Formulierung in »De servo arbitrio",WA 18, 685,11ff, "verbum et cultum quo Deus nobis cognitus est et nobiscum habet commercium «, in dessen weiterem Kontext Luther beschreibt, dass Gott uns überhaupt nur im Wort zugänglich ist, weil er sich darin (als gnädig und barmherzig) bestimmt ("definivit sese", Z. 23) hat.

${ }^{10}$ Ringleben, Gott im Wort, 451. 
menschlichen commercium ${ }^{11}$ und sagt sich in diesem aus als Verb einer verheissungsvollen Anrede; und zwar immer wieder neu. ${ }^{12}$

\section{b. Wort und Schrift}

Diese formale Beschreibung des Glaubens wird besonders anschaulich in der Beobachtung des Lesevorgangs der heiligen Schrift, wobei der Aspekt der Zeitlichkeit noch einmal klar beleuchtet wird. Zugleich nähern wir uns mit ihm der praxis pietatis des Gebets. Schrift, so Luther bei Ringleben, ist eigentlich Stimme und Lesen eigentlich Hören; in diesem Sinne verwickelt es in das eben genannte Gespräch. Mündlich wird der Text (wieder) indem durch die graphische Fixierung des Gesagten dennoch die Stimme vernehmbar ist, die spricht, und die Ringleben in Anschluss an Derrida als Phänomen absoluter Präsenz beschreibt. ${ }^{13}$ Den Text als Stimme wahrzunehmen, bedeutet ihn zu hören - und das heisst im Fall des NT der vox Christi als Anrede inne zu werden. Insofern ist »das Lesen [...] erst als echtes Hören lebendiger Vollzug von Sprachlichkeit. «14 Bei der Kennzeichnung der Sprachlichkeit des schriftlichen Wortes kommt Ringleben auf drei Punkte zu sprechen, die die Oralität kennzeichnen: erstens erfülle sich durch sie die extrinsische Glaubensstruktur; zweitens stelle sich beim Lesen bzw. Hören der unmittelbare lebendige Gottesbezug ein; und drittens ereigne sich im Hören der Kairos der Präsenz: »Das [beim Lesen, J.B.] gehörte Wort,

\footnotetext{
${ }^{11}$ Vgl. dazu auch in Kapitel XI. Wort und Geist, wo entfaltet wird, wie Menschenwort und Gotteswort zusammen sind: „Die stimmliche Verlautbarung des Menschen beim Sprechen, sein sinnlich-äußerliches Reden in Worten, kann also die Weise sein, dergemäß wir saudimus Spiritum sanctum Äußerlichkeit die menschliche Natur in seine Gegenwart hineingenommen und aufgehoben hat, sie darin beherbergt (colligere) und für uns da sein läßt, hat Gott in unbegreiflicher und barmherziger Herablassung (placuit) es ermöglicht, sut possemus eum apprehendere r. Ringleben, Gott im Wort, 486f.

${ }^{12}$ Damit soll die Beständigkeit des Glaubens als Herzensvertrauen bzw. die Gewissheit des Glaubens nicht ignoriert werden, nur liegt auf ihr hier nicht der Fokus.Vgl. dazu Ringleben, Gott im Wort, 459f: "Der Gottes Macht im Menschenwort der h. Schrift oder der Predigt innewerdende Glaube verhält sich mithin genuin sprachlich, wenn er im vergehenden Laut (und gerade nur in ihm) doch zugleich die bleibende Kraft vernimmt«. S. auch 469.Vgl. auch Dietrich Korsch, Glaube und Rechtfertigung, in: Albrecht Beutel (Hg.), Luther Handbuch, Tübingen ${ }^{2} 2010,372-381$, hier 373, der auf die (notwendig abstrakte) Struktur des Glaubens als "Grund des menschlichen Selbstseins" abhebt.

${ }^{13}$ Zweifellos ist der Anschluss diskussionswürdig insofern es bei Derrida ja gerade um die Kritik an derVerbindung von Oralität und Präsenz geht.

${ }^{14}$ Ringleben, Gott im Wort, 423.
} 
das uns anredet, gewährt uns Zeit, gerade indem es uns von uns weg an Gott verweist." An dieser Stelle macht er zudem einen Verweis auf Hamanns Satz: »ich (weiß) von keinen ewigen Wahrheiten, als unaufhörlich Zeitlichen «. ${ }^{15}$

An diesem Satz sollen drei Punkte noch einmal kurz herausgestellt werden. Zum ersten dieVerknüpfung von Selbstsein und Zeit, die in invertierter Logik in den Blick kommt. Ist doch das übliche Empfinden, Zeit zu haben ginge mit der Kontrolle über die eigene Zeit einher. Dann ist es "meine Zeit«, wenn ich verfügen kann, wo und mit was ich sie verbringe - eben autonom über sie zu bestimmen und nicht heteronom anderen Rhythmen unterworfen zu sein. ${ }^{16}$ So lässt sich formal festhalten: Wer ich bin, hat damit zu tun, womit ich meine Zeit verbringe. Und wenn ich Zeit verliere, ist damit - wie im Wort von der (ablaufenden) Lebenszeit anklingt - auch der empfindliche Konnex von Zeit und Selbst berührt.

Auf diesem Hintergrund klingt hier die - auf verschiedenen theologischen und philosophischen Registern durchzuspielende Dialektik des Selbstseins an, die darin besteht, dass gerade in dem Moment der Selbstenthobenheit oder stärker: dem Moment der Selbstvergessenheit das Selbst zu sich kommt: Gewährung von Zeit gerade durch ihren Entzug des an Zeit begrenzten Menschen. ${ }^{17}$ Zeitlosigkeit ("weg von uns") kommt in der von Ringleben dargestellten Leseerfahrung also zusammen mit erfüllter Zeit und konvergiert im Begriff der Präsenz.

\section{c. Wort und Gebet}

Mit diesen Einsichten zu Wort, Glaube und Zeit ist Wesentliches vom Gebetsverständnis Luthers bereits benannt und deswegen soll die explizite Übertragung auf das Gebet an zwei ausgewählten Textbeispielen knapp erfolgen.

Ist der Glaube, des Herzens Vertrauen und Hängen an Gott, wesentlich sprachlich verfasst, so findet das seinen Ausdruck auch darin, dass Luther in seiner Gebetspraxis vor allem auf die Texte der Bibel und der kirchlichen Bekenntnisse Bezug nimmt. Das führt er

\footnotetext{
15 Ebd., 426.

${ }^{16}$ Man denke nur an diesen Satz mit seinen allfälligen Varianten: »Der oder das stiehlt mir die Zeit! «

17 Paradigmatisch steht dafür das Bild des in Selbstvergessenheit spielenden Kindes, welches in seinerVersunkenheit einen starken Eindruck vom einfachen So-Sein eines Menschen gibt und das gesättigte Wort der Seligkeit (vgl. Spr 8,30) evozieren kann, womit die höchste Möglichkeit des Vergessens aufgerufen sein dürfte.
} 
beispielsweise in einem Trostbrief an seinen Freund Peter Balbier aus. ${ }^{18}$ Dieser befindet sich in einer Gebetskrise und Luther schreibt, dass er Empfindungen der Unlust und der Herzenskälte gut nachempfinden könne. In diesen Fällen, so Luther, geht er entweder in seine Kammer oder zum gemeinsamen Gebet in der Kirche und nimmt dort vorzugsweise den Psalter, das Vaterunser, das Credo oder auch die Zehn Gebote als "Feuerzeug« hervor, um das verdrossene kalte Herz zu entzünden. ${ }^{19}$ Es geht darum, dass das Herz - gerade durch die Reibung an den Texten von ausserhalb, die der eigenen Stimmung widerstehen $-\mathrm{zu}$ sich selbst kommt und zum Gebet frei und erwärmt wird..$^{20}$ Dabei haben das Gebot und die Verheissung Gottes erstens die fundamentale Funktion, das Gebet überhaupt zu begründen. ${ }^{21}$ Sodann nehmen sich die Texte der Bibel und der Tradition im konkreten Verlauf des Betens als Hilfen aus, ${ }^{22}$ die als Besinnungsmittel selbständig zu gebrauchen und auszuwählen sind und dann in den Hintergrund treten, wenn es dazu kommt, dass der Geist selbst zu reden anfängt. Luther empfiehlt:

$»[\ldots]$ ob der Heilige geist unter solchen gedancken keme und anfienge jnn dein hertz zu predigen mit reichen erleuchten gedancken, so thw [tue] jm die ehre, lase diese gefasste dancken [Gedanken] faren, sey stille und hoere dem zu, ders besser kann denn du, Und was er predigt, das merck und schreibe es an [auf], so wirstu wunder erfaren (wie David sagt) im Gesetz Gottes. "23 $^{23}$

In diesem Moment ist an das Phänomen der Stimme und der Präsenz zu erinnern, welches oben im Zusammenhang derVersprachlichung des Textes erwähnt wurde: beim Lesen kommt es zum Hören einer

\footnotetext{
${ }^{18}$ Martin Luther, Eine einfältige Weise zu beten für einen guten Freund [1535], WA $38,358-375$.

${ }^{19}$ Ebd., 373, 6.

${ }^{20}$ Ebd., 360, 1f; 372, $27 f$.

${ }^{21}$ Ebd., 360, 6-11; vgl. Bernhard Mutschler, Ein Reden des Herzens mit Gott. Martin Luther über das Gebet, in: NZSTh 49 (2007), 24-41, hier 28, der neben den beiden Gründen von Gebot und Verheissung auch noch die Not erwähnt, und Gunnar Wertelius, Oratio continua. Das Verhältnis zwischen Glaube und Gebet in der Theologie Martin Luthers, Lund 1970,108-110, 120, der die Zwiefältigkeit des Gebots zu beten als Ermutigung und Befehl beleuchtet und die Bedeutung der Gewissheit, die die Verheissung schaffen will, betont.

${ }^{22}$ Luther formt ein richtiges Instrument aus seinem Durchgang durch Vaterunser und Dekalog, indem er zu jedem Abschnitt jeweils lehrhafte, dankende, beichtende und bittende Betrachtungen von sich selbst vor Gott anstellt und diese vierfache Auffächerung ein "gevierdes oder ein vierfach gedrehtes krentzlin" $(364,31)$ nennt. Vgl. dazu Mutschler, Reden des Herzens, 24-41, hier 33f.

${ }^{23}$ Luther, Einfältige Weise, WA 38,366,11-15.
} 
Stimme, die predigt, die anspricht und hier das eigene Sinnen und Nachdenken zugunsten des Zuhörens zum Schweigen bringt. ${ }^{24}$ Was oben zur Wortförmigkeit des Glaubens gesagt wurde, spiegelt sich hier wieder: die Worte garantieren zum einen die inhaltliche Bestimmtheit und richten den Beter der Sache nach aus.Vor allem aber ist das Ergriffen- oder Entzündetwerden durch die Worte wichtig, was Luther mit dem Heiligen Geist, der ins Herz predigt, anspricht. ${ }^{25}$ Diese Unterscheidung tritt noch stärker hervor in der Auslegung des Vaterunsers von 1519, in der Luther zu Beginn drei Weisen zu beten bestimmt, von denen die ersten beiden (aus blossem Gehorsam und ganz ohne Gehorsam) deutlich gegenüber der dritten abfallen, die mit Andacht des Herzens betet. ${ }^{26}$ Gerade in dieser dritten Art, obwohl die Beterin dabei besonders wenig Worte macht oder sie gar ganz fahren lässt ${ }^{27}$, kommt die Wortbezogenheit erst zu ihrer vollen Wahrheit: sie besteht darin, das Herz zu Gott emporzuheben und $\mathrm{zu}$ bewegen und damit die Worte $\mathrm{zu}$ »irem ampt und frucht [zu] gebrauch[en] «28, welche in nichts anderem bestehen, als ein immer noch grösseres Vertrauen zu Gott zu wirken. Letztlich zielen sie auf das Gebet "innerlich mit dem hertzen, das got am meysten anligt, unnd auch dasselb allein ansicht und sucht. $\mathbb{1}^{29}$

Was den Aspekt der Zeitlichkeit angeht, so schärft Luther selbst immer wieder die Bedeutung der Regelmässigkeit ein. Auch wenn durchaus gilt, dass die richtigen, d.h. auf Gott gerichteten Gedanken jegliches Werk zum Gebet machen, so dürfe das doch nicht dazu führen, das eigentliche Werk des Gebets ausser Acht zu lassen. ${ }^{30}$ Und

\footnotetext{
${ }^{24}$ Dieses Schweigen müsste man genauer charakterisieren, da es wohl eher eine Qualität des sich einstellenden Gedankenflusses meint als ein völliges Versiegen dessen.Vgl. hierzu Claudia Edith Kunz, Schweigen und Geist. Biblische und patristische Studien zu einer Spiritualität des Schweigens, Freiburg 1996, 20-37. Luthers Erfahrung würde sich nach der von Kunz vorgelegten Grammatik des Schweigens wohl am besten als Stille innerhalb eines verbalen Schweigens verstehen lassen: ein Schweigen, in dem der Logos gleichwohl aktiv beschäftigt ist und innerhalb dessen sich eine Verschränkung von sinnlicher und diskursiver Wahrnehmung mit dem Herzen ereignet.

${ }^{25}$ Vgl. zum Hl. Geist als Symbol für die Selbstunterscheidung Gottes am Menschenwort Ringleben, Gott im Wort, 485-489.

${ }^{26}$ Martin Luther, Auslegung deutsch des Vaterunsers für die einfältigen Laien [1519], WA 2, 80-130, 81, 29-82, 21.

${ }^{27}$ Ebd., 82, 20f.

${ }^{28}$ Ebd., 85, 32.

${ }^{29}$ Luther geht hier davon aus, dass das Herz irgendwann durchaus auch ohne Wort derart "fliegen", d.h. ohne in eigene Gedankenwirrnisse zu verfallen sich im Gebet auf das eigene Herz verlassen könne (85). Im Kontext wird klar, dass er das für eine seltene Möglichkeit hält; der grundlegende Wortbezug gilt ohnehin.

${ }^{30}$ Luther, Einfältige Weise, WA 38,359,10-34.
} 
damit ist eben jener Umgang mit den Worten gemeint, die ein Feuer entzünden, das es gilt stets am Brennen zu halten; von daher erscheint die Rhythmisierung des Gebets entlang des (Beginns und Endes des) Tages folgerichtig. ${ }^{31}$ Eingebettet in diesen festen Rahmen sind sodann unregelmässige, oft gesprochene Stossgebete, die ebenfalls »hitzig sein sollen. ${ }^{32}$ Für mein Interesse ist hier noch einmal gesondert zu bemerken, dass Luther gerade dann auf dem Gebet besteht (und von den Werken, die ja von guten Gedanken an Gott begleitet werden können, abgrenzt), wenn die Zeit knapp wird; also auf Zeitknappheit mit einer Gewährung von Zeit (für das Gebet) zu reagieren um, so könnte man weiterführen, auch sich selbst wieder präsent zu werden. So erscheint es nicht überraschend, dass er am Ende dieses Abschnitts zum ersten Mal die oben bereits zitierte Formulierung bringt, dass das Herz im Gebet zu sich selbst kommt und frei wird für das Wort, welches ihm darein gesprochen wird. Insofern erscheint möglich, den eingangs zitierten Satz »Das Gebet ist das Herz des Glaubens" so zu verstehen, dass das Gebet nicht nur der Ort der Belebung des Glaubens, ${ }^{33}$ sondern auch der Entstehung von Zeit ist.

\section{Das Gebet im Kontinuum des Bewusstseins - Gefühl und Gebet bei Schleiermacher}

Während bei Luther der Zusammenhang von Wort und Glaube fast von selbst auf das Gebet überleitete und die Bedeutung des konkreten Wortes in der Gebetspraxis sich auch in einer gewissen Umfänglichkeit der Darstellung zur Geltung brachte, so werde ich bei Schleiermacher direkt beim Gebet einsetzen und von da aus nur hin und wiederVerweise auf seine Glaubenskonzeption geben.

\footnotetext{
${ }^{31}$ Ebd., 359, 4f; vgl. BSLK 547, 33-549, 16, wo es darum geht, dass der Umgang mit dem Wort täglich neu eingeübt werden muss, da der Geist an ihm nur jeweils gegenwärtig "immer neue" und klarere Einsicht und Andacht zu wirken vermag.

${ }^{32}$ Luther, Einfältige Weise, WA 38, 372, 31.

${ }_{33}$ Martin Luther, Predigt am Sonntag Vocem Iocunditatis, nachmittags [1. Mai 1531], WA 34/1, 395,13f: "Habes not und gewaltige ursach per eius praeceptum. Qui non orat, mit der zeit wird er fidem verlieren."So zitiert bei Mutschler, Reden des Herzens, 29, Anm. 29.
} 


\section{a. Denken an Gott}

Auch Schleiermacher verortet das Gebet in der "Mitte " des Glaubens, wie es in jenem bekannten ersten Satz aus seiner Predigt über Mt 26,36-46 heisst: „Fromm sein und beten, das ist eigentlich ein und daßelbige. ${ }^{34}$ Damit ist die Entgrenzung des Gebets bzw. die Loslösung von spezifischen Gebetszeiten programmatisch vorgegeben, denn es gehe darum, so Schleiermacher weiter, "[a]lle Gedanken von einiger Wichtigkeit, die in uns entstehen, mit dem Gedanken an Gott in Verbindung zu bringen" - und das in allen Lebenslagen. Deshalb wird schon hier deutlich, dass Schleiermacher einen zeitlich entgrenzten Gebetsbegriff im Blick hat und das Gebet im Folgenden als »Beten ohne Unterlaß« bestimmt; genau dies mache nämlich das "Wesen der wahren Frömmigkeit « aus. ${ }^{35}$ So sehr es aber zeitlich ungebunden ist, so bestimmt fixiert Schleiermacher die Zielrichtung dieses Betens, wie nun zu zeigen ist.

In seinen Ausführungen über das Gebet in der Glaubenslehre entfaltet er seine in der Predigt grundgelegten Einsichten dazu im Zusammenhang seiner dogmatischen Systematik. ${ }^{36}$ In ihr weist er dem Gebet seinen Ort im zweiten Hauptstück der Lehre von der Kirche zu, deren Aufgabe darin besteht, das durch Jesus Christus gestiftete neue Gesamtleben weiter auszubilden, bis schliesslich die ganze Welt davon erfasst ist: Es geht, anders gesagt, um die Ausbreitung des Reiches Gottes auf Erden. ${ }^{37}$ Dabei wird der Kirche, durch Wirkung des Heiligen Geistes, immer wieder bewusst, wie weit sie noch von ihrem ihr gegebenen Ziel entfernt ist und insofern "schwebt" sie "zwischen Gegenwart und Zukunft«. ${ }^{38}$ Auch hier findet sich nun wieder die Formulierung des Denkens an Gott und zwar so, dass es in Bezug auf das schon Gewesene zu Dank oder Ergebung wird (je nachdem, ob es freudig oder leidvoll bejaht wird); in Bezug auf das Kommende, von dem jeder eigene Wunschvorstellungen oder auch ein »Vorgefühl $\aleph^{39}$ hat, wird es zum Bittgebet:

\footnotetext{
${ }^{34}$ Friedrich Schleiermacher, Die Kraft des Gebetes, in so fern es auf äußere Begebenheiten gerichtet ist, in: Predigten. Erste bis Vierte Sammlung (1801-1820) mit den Varianten der Neuauflagen (1806-1826), Günter Meckenstock (Hg.), KGA III/I, Berlin/Boston 2012, 25-38, hier 25, 4. Vgl. zur werkgeschichtlichen Einordnung Rolf Schäfer, Zu Schleiermachers Lehre vom Gebet, in: Michael Pietsch/Dirk Schmid (Hg.), Geist und Buchstabe. Interpretations- und Transformationsprozesse innerhalb des Christentums. FS Günter Meckenstock, Berlin 2013, 361-381.

${ }^{35}$ Schleiermacher, Kraft des Gebetes, KGA III/I, 25, 4-10.

${ }^{36}$ Schäfer, Lehre vom Gebet, 367.

${ }^{37}$ Ebd., 368.

${ }^{38}$ Schleiermacher, Glaubenslehre, $\$ 146$, KGA I/XIII,2, 418, 5.

${ }^{39}$ Ebd., 419, 4.
} 
»innige Verbindung des auf das beste Gelingen gerichteten Wunsches mit dem Gottesbewußtsein. $\star^{40}$ Allerdings erweisen sich diese drei möglichen Formen des Gebets in Bezug auf das grosse Ziel nur als "Durchgangspunkte ${ }^{41}$, die die Kirche am besten hinter sich liesse: denn das Reich Gottes wird ohne Zweifel schliesslich gänzlich aufgerichtet werden, so dass aus den Bitten Grund zu Dank und Ergebung werden wird, Ergebung und Dank in diesem (uns nicht im Einzelnen einsichtigen) Heilsverlauf aber auch noch die Plätze tauschen können. Deswegen sei es ratsam, dass die Kirche, "nur die untrügliche Gewißheit des endlichen Erfolgs festhaltend in der Freude an Gott zur vollkommenen Ruhe gelangt. ${ }^{42}$ Entsprechend kann Schleiermacher es auch nicht gutheissen, wenn sich jemand rühmen wollte, regelmässig oder gar häufig zu beten. ${ }^{43}$

Eben diese Zielformulierung der Ruhe findet sich auch in der eben angesprochenen Predigt. Nicht nur sollte man das beim Beten unterlassen, was "unserer Ruhe nachtheilig ist ${ }^{44}$, nämlich in dem Sinne an die Erhörung der Gebete zu glauben, dass sie bei Gott etwas bewirken. Das ist ausgeschlossen, denn eher würden Himmel und Erde vergehen, als das Gott etwas an seinem Ratschluss ändere. ${ }^{45}$ Vielmehr soll man sich ein Beispiel an dem Beter Jesus Christus nehmen, der auch in seinem schwachen Moment im Garten Gethsemane, als er Gott dringlich um etwas bat, noch ein Vorbild für uns ist: Im Lauf seines dreimaligen Betens in jener Nacht mässigte der Gedanke an seinen Vater im Himmel nämlich zunehmend seinen Wunsch, verschont zu werden, und

wals er zum dritten Mal gebetet hatte, war alle Besorgniß und alles Zagen verschwunden, er hatte keinen Wunsch mehr, sondern [...] mit gelaßenem Gemüth, mit frommer Tapferkeit ging er seinem Verräther entgegen. Sehet da, das ist die Wirkung, welche ein solches Gebet hervorbringen soll. «t

\footnotetext{
${ }^{40}$ Ebd., 418, 12-14.

${ }^{41}$ Ebd., 418, 20-27.

${ }^{42}$ Ebd.

${ }^{43}$ Schleiermacher, Kraft des Gebetes, KGA III/I, 36, 22-37, 33.

${ }^{44}$ Ebd., 26, 10.

${ }^{45}$ Ebd., 30,17-20.

${ }^{46}$ Ebd., 31, 20-26.Vgl. auch folgende Formulierung im Rahmen der Zusage, dass Gott unsere kleinen Anliegen als Teil des grossen Ganzen berücksichtigen wird: »so entsteht Ruhe, denn was uns auch begegne, es muß Gutes herauskommen, und so ruft endlich das stillgemachte und besänftigte Herz:Vater, es geschehe dein Wille." (KGA III/I, 33, 2-5) Zur Nähe zwischen Pietismus und Quietismus s. Robert Leuenberger, Zeit in der Zeit. Über das Gebet, Zürich 1988, 132-136.
} 
Da es allerdings unter den Bedingungen menschlicher Subjektivität nicht möglich ist, das Wünschen und Vorstellen aufzugeben, so soll ruhig »ohne Unterlaß« weitergebetet werden, was eben heisst, diese Tätigkeit des Bewussteins mit dem Gottesbewusstsein zu verbinden. ${ }^{47}$

Diese (eher) formalen Beschreibungen sind nun aufs engste mit der inhaltlichen Bestimmung des Betens verbunden, welche Schleiermacher aus der Formulierung gewinnt, von der er im Folgenden ausgeht: »im Namen Jesu beten «. ${ }^{48}$ Damit ist die Bezogenheit jeglichen Betens auf das Reich Gottes gemeint, was wiederum die Kirche ins Spiel bringt, insofern sie diesem als konkrete geschichtliche Form zur Durchsetzung verhilft und das bedeutet für in Frage kommende Inhalte eines wahren Gebets, das es nur um solches gehen kann, »ohne welches ein Wiedergebohrener nicht könnte in dem Stande der Heiligung erhalten werden, oder ohne welches das Reich Christi nicht könnte Bestand und Fortgang haben. ${ }^{49}$

\section{b. Glaube und Gebet}

Der dabei von Christus als Erhörungsbedingung geforderte Glaube beziehe sich dabei nicht im Speziellen auf die Erhörung, sondern vielmehr auf den (rechten) Glauben an Gott überhaupt. Der vielfach beobachtete Zusammenhang von Gotteslehre und Gebet ist in Schleiermachers systematischer Konzeption eminent: um ein rechtes Verständnis vom Gebet und seinen Grenzen zu gewinnen, müsse man bedenken, zu wem man betet. Und so wendet Schleiermacher sich der Beschreibung Gottes zu: Gott ist ein »unveränderliche[s] Wesen", er ist "der Unerforschliche«, "der Alleinweise" und "der Gütige $\star^{50}$, was er in der Glaubenslehre dann folgendermassen als »erste Grundvoraussetzung" zuspitzt: nämlich »daß es kein Verhältnis der Wechselwirkung giebt zwischen Geschöpf und Schöpfer«. ${ }^{51}$ Und daher bedeutet Glaube "Glauben an die Unvergänglichkeit und den alles andre sich unterordnenden Werth des von ihm gestifteten

\footnotetext{
${ }^{47}$ Schleiermacher, Glaubenslehre, $\$ 146$, KGA I/XIII,2, 418,26-36.

${ }^{48}$ Ebd., \$147, KGA I/XIII,2, 421, 435.

${ }^{49}$ Ebd., 423,15-19.

${ }^{50}$ Schleiermacher, Kraft des Gebetes, KGA III/I, 32,1-34.

${ }^{51}$ Schleiermacher, Glaubenslehre, \$147, KGA I/XIII,2, 423, 4f. Zur Grundlegung dieses Satzes im Theorem des schlechthinnigen Abhängigkeitsgefühls s. Schäfer, Lehre vom Gebet, 376.Vgl. auch Dietz Lange, Neugestaltung christlicher Glaubenslehre, in: Ders. (Hg.), Friedrich Schleiermacher.Theologe - Philosoph - Pädagoge, Göttingen 1985, 85-105, hier 97.
} 
Reiches Gottes «. ${ }^{52}$ Aus diesen Sätzen gewinnt Schleiermacher die Fassung eines Gebets, das seine Hörer zugleich vertrauens- und ergebungsvoll stimmen soll und sie vor der "peinlichen Ungewißheit «s die im Gottesverhältnis durch einen ungeläuterten Erhörungsglauben entstehen könnte, von Anfang an bewahrt. An dieser Stelle sei auf die deutliche Differenz zu Luther nur hingewiesen: während bei Luther der Glaube an dem (sich) der Zeit unterworfenen Wort hängt und nur durch den immer wiederholten Umgang mit den Worten zur Gewissheit des Glaubens kommt, so wird das Gebet bei Schleiermacher als ein Tun verstanden, dass sich der Zeit geradezu entzieht, indem es, wie ein Medium, das Zeitliche so in Kontakt mit dem unwandelbaren ewigen Gott in Verbindung bringt, dass es sich dessen Zeitlosigkeit unterordnet und den Wunsch in Dank oder Ergebung verwandelt. ${ }^{54}$

Allerdings, so sei abschliessend noch erwähnt, spielt in der Praktischen Theologie die Bedeutung der Worte im Sinne extern zukommender Reizung doch eine Rolle und zwar im Zusammenhang mit der Predigt. In der »Theorie des Gebets im Cultus" bedenkt Schleiermacher die Stellung, die Form und den Inhalt des Gebets im Gottesdienst. ${ }^{55}$ Leitend ist hier seine Vorstellung vom gemeinsamen Gebet als Ausdruck einer gemeinsamen religiösen Stimmung, die zu stimulieren und zu vermitteln der Liturg als Aufgabe hat. Dabei muss er insbesondere auf den Ausgleich zwischen der Gesamtstimmung und den Stimmungen der einzelnen Gottesdienstbesucher achten, welche weit auseinanderfallen können; auch wenn der Sache nach das Gebet des Einzelnen und das kollektive Gebet dasselbe sind: »erhöhte[r] Ausdruck des Bewußtseins der Abhängigkeit von Gott. ${ }^{56}$ Für das Gebet am Anfang des Gottesdienstes kann er sich für seine Aufgabe nur auf die allgemeine christliche Religiosität der Besucherinnen sowie den Gesang verlassen. In seiner Predigt aber kann er die gemeinsame Stimmung gezielt fördern bzw. formen. Insofern aber das Gebet »das Maximum der Äußerung der religiösen Stimmung« ist, die Rede aber eben diese maximal fördern soll, so

\footnotetext{
${ }^{52}$ Schleiermacher, Glaubenslehre, $\$ 147$, KGA I/XIII,2, 423, 24-26.

${ }^{53}$ Schleiermacher, Kraft des Gebetes, KGA III/I, 26,11f.

${ }^{54}$ Vgl. dazu Lange, Neugestaltung, 100, wo er auf Schleiermachers Christusbild eingeht: ein "Mann, der bei aller menschlichen Zuwendung zu den Leidenden doch selber dem Alltäglichen ganz entrückt erscheint, ein[ ] feste[r] Charakter ohne inneren Bruch».

${ }^{55}$ Friedrich Schleiermacher, Die praktische Theologie nach den Grundsätzen der evangelischen Kirche im Zusammenhange dargestellt, in: Jacob Frerichs (Hg.), Friedrich Schleiermachers sämtliche Werke I/13, Berlin 1850, 187.

${ }^{56}$ Ebd., 188.
} 
»ist das Gebet [insoweit] noch ein Bestandtheil der religiösen Rede und aus ihr hervorwachsend. $\ll^{57}$

Wie bei Luther so geraten auch hier Gebet und Predigt in Nähe zu einander; während bei Luther aber der Heilige Geist beim Beten anfing ins Herz zu predigen, so ist es hier der Prediger, welcher vermittels seiner Rede die Herzen der Hörer erhebt, in Stimmung bringt und seine Rede schliesslich in Gebetsworte münden lässt. Damit wechselt er erstens seine Sprechrichtung, indem er jetzt nicht mehr zur Gemeinde, sondern zu Gott spricht, und weitet seine Sprechrolle zweitens so, dass er zum stellvertretenden Organ des gemeinschaftlichen Gebets aller wird.

\section{c. Vergleichendes Zwischenfazit}

Am Ende dieser beiden ersten Abschnitte zu Luther und Schleiermacher sollen die Beobachtungen kurz noch einmal vergleichend festgehalten werden.

Zunächst ist zu betonen, dass Schleiermacher sich dem Gebet hauptsächlich als einem locus communis widmet, jedoch auch auf das Gebet des Einzelnen eingeht. Hier ist dann zu beobachten gewesen, dass bei beiden Autoren die Rhetorik der Innerlichkeit eine bedeutende Rolle spielt, aber wenn man so möchte, in einer völlig anderen Färbung - während dort im Herzen ständig ein Feuer entfacht werden soll und Betrachtung, Bekenntnis, Dank und Bitte sich abwechseln wie die Perlen einer Schnur, geht es hier um beständige Erhebung der Gedanken, die eine Haltung stiller Ergebung formen und in die geklärte Ruhe einer friedvollen Seele münden. Während bei Luther die Worte der Orientierungspunkt ständiger Vergewisserung und Entzündung sind, so tritt bei Schleiermacher höchstens die Rede des Prediger als Stimulans der allgemeinen Stimmung der gottesdienstlichen Gemeinde als bedeutsam für das Schlussgebet im Gottesdienst auf. Es sind vielmehr die Begebenheiten des Alltags, die Anlass zum Gebet werden - allerdings eher nicht im Sinne von Stossseufzern, sondern als Verbindung der konkreten Gedanken und Gefühle mit dem Gedanken an (den unveränderlichen) Gott, dessen Reich die Bedeutung des Einzelnen und seiner leiblich-konkreten Existenz auf ein solches Mass reduziert, dass sogar dessen Verlust nicht schliesslich auch den ergebensten Dank zeitigen würde. Und während dort das Gebet als Pulsgeber des Glaubens und, indem es vor allem Geschehen von Präsenz ist, als Ort der Entstehung von Zeit in Blick kam, indem es Zeit beansprucht, so flicht sich das Ge-

${ }^{57}$ Ebd., 195. 
bet hier als ein quasi lautloses Aufblicken zu Gott im Kontinuum des Bewusstseins in den ungehindert fortfliessenden Strom des alltäglichen Tagesablaufs. Demgegenüber erscheinen die spezifischen Dankoder Bittgebete nur als vorübergehende Durchgangspunkte. Bei beiden Autoren schliesslich spielt die gedankliche Dimension eine wichtige Rolle, allerdings wiederum in sehr unterschiedlicher Weise. Bei Schleiermacher hängt das Gebet am stets tätigten Bewusstsein des Subjekts, das keiner Regulierung oder Anstrengung bedarf, um sich mit dem Gedanken an Gott zu verbinden. Das Bewusstsein ist der Ort des Gebets schlechthin. Bei Luther hingegen gleicht das diskursive Nachdenken anhand von Texten einem Feuerstein, mit dem das kalte Herz erwärmt werden soll: um Worte zu finden und sich von ihnen finden zu lassen.

\section{Das immerwährende Herzensgebet}

Das Problem, von dem ich ausgegangen war, lässt sich nun noch einmal zugespitzt formulieren, verlässt damit die Ebene des Textreferates und ruft theologische Horizonte auf, die jetzt aber in weiter Ferne belassen werden müssen. Auf der einen Seite, so könnte man sagen, steht ein Verständnis von Zeit bzw. Gebet, das sich am Ereignischarakter festmacht: Das Gebet, insofern es Gespräch ist, ist eingebettet in den Wechsel menschlichen Zeiterlebens und damit rhythmisch koordiniert. Mit der Wortförmigkeit ist die leibliche, konkrete, vergängliche Gestalt dieses Gebets gemeint, die der Zeit preisgegeben ist. Und nur unter diesen Bedingungen kommt es zu Gewährung von Zeit, zu Präsenz durch Anrede, die diese Bedingungen jeweils und immer wieder neu momenthaft aufsprengt. Der Kairos durchbricht die Zeit.

Auf der anderen Seite steht ein Verständnis von Zeit bzw. Gebet, das sich an der Monotonie orientiert und sich die konkreten leiblichen Gegebenheiten des Lebens zu verwechselbaren Anlässen des Andenkens an Gott dienen lässt. Sie alle sind nur Stationen auf der Geraden, deren Anfangspunkt die Geburt des Erlösers markiert. Ihr entlang fliesst nun das Leben der Kirche und schreitet unaufhaltsam und gelassen ihrem feststehenden Erfolg entgegen. Insofern ist die Heilszeit ein Kontinuum, das leicht über den Wechselfallen des Lebens schwebt und diese im Medium des Gebetes einstimmt auf den seligen Frieden der Ewigkeit, wo Zeit nicht mehr ist. 


\section{a. Gebet als Weg}

In der Gestalt des immerwährenden Herzensgebets, so war die Ausgangsthese, könnte sich eine Versöhnung dieser beiden Typen andeuten lassen. Dies soll nun in wenigen Worten geschehen.

Das Herzensgebet ist in der asketischen Mönchsbewegung des frühen Christentums verwurzelt und vor allem in den orthodoxen Kirchen in Form mündlicher Vermittlung verbreitet worden. Im Westen hat diese Tradition vor allem durch die Aufrichtigen Erzählungen eines russischen Pilgers Verbreitung seit der zweiten Hälfte des 20. Jahrhunderts gefunden. ${ }^{58}$

Es geht in diesem Gebet, allerdings mit weit reichenden theologischen Implikationen besonders der orthodoxen Anthropologie, darum, mittels des unermüdlichen Wiederholens eines einzelnen Satzes "Herr Jesus Christus, Sohn Davids, erbarme dich meiner" (vgl. Lk 18,38 par.) bzw. allein des Namens Jesu Christi eine ununterbrochene Wahrnehmung von Gottes Anwesenheit im eigenen Herzen sowie in allem Geschaffenen überhaupt zu erlangen. Dies ist gleichbedeutend mit der im Hesychasmus - jener Frömmigkeitsausprägung, die dazu anleitet, den inneren Frieden in Stille und Einsamkeit zu suchen - ausgeprägten Vorstellung des sich-in-Gott-Versenkens bzw. der Einswerdung mit Gott:

"Hesychia ist Stillesein des Geistes und der Welt, Vergessen des Niedrigen, geheimnisvolles Erkennen des Höheren, das Hingeben der Gedanken an etwas Besseres, als sie selber sind. So schauen die, die ihr Herz durch solch heiliges Schweigen (Hesychia) gereinigt und sich auf unaussprechliche Weise mit dem alles Denken und Erkennen übersteigenden Lichte vereinigt haben, Gott in sich selbst wie in einem Spiegel. ${ }^{59}$

Diese Schau mit dem Herzen setzt, wie im Zitat anklingt, eine Reinigung voraus, an die der Mensch durch die Teilnahme am Leben der Kirche selbsttätig mitwirken kann $^{60}$ und die als dreigestufterWeg in dieser von neuplatonistischem Gedankengut gesättigtenTradition

\footnotetext{
${ }^{58}$ Zur geschichtlichen Entwicklung s. Christoph Klein, Das grenzüberschreitende Gebet. Zugänge zum Beten in unserer Zeit, Kap.VI.3 Das Herzensgebet, 105-112, 106-108. Die Erzählungen in folgender Ausgabe: Aufrichtige Erzählungen eines russischen Pilgers. Erste vollständige deutsche Ausgabe. Herausgegeben und eingeleitet von Emmanuel Jungclaussen, Freiburg/Basel/Wien ${ }^{2} 1974$.

${ }^{59}$ Gregor Palamas zitiert bei Jungclaussen, Aufrichtige Erzählungen, 11. Zur Etymologie und biblischen Verwendung des Begriffs s. Kunz, Schweigen und Geist, 23f.

${ }^{60}$ Dazu gehören u.a. das regelmässige Lesen der Schrift und die Teilnahme an den Sakramenten. Von besonderer Bedeutung ist wohl aber das Bemühen um die innere
} 
zahlreiche Ausprägungen gefunden hat. ${ }^{61}$ Immer geht es darum, als Mensch in allen Konstellationen der eigenen Lebensvollzüge geistlich bestimmt zu sein. ${ }^{62} \mathrm{Zu}$ diesem Ziel, so die Annahme, führt ein Weg, so dass Gebet hier vor allem als Prozess in den Blick kommt. Dieser wiederum vollzieht sich in mehreren Schritten. ${ }^{63}$ Zunächst wird das Gebet mündlich aufgesagt und zwar in sich steigernder Frequenz. Der Lehrer des Pilgers weist ihn, das Gebet zunächst 3.000 Mal am Tag, schliesslich, über Wochen hinweg, 12.000 Mal am Tag zu sagen. ${ }^{64}$

Von ihm eigens gewidmeten Gebetszeiten weitet sich das Gebet, immer noch als mündliche Wiederholung, auf die übrigen Stunden und Tätigkeiten aus. Nach einer gewissen Zeit fängt der sog. Pilger wieder an zu wandern und dabei das Gebet stets leise vor sich hin zu murmeln, bis er merkt, dass während des mündlichen Gebets »das Gebet ganz von selbst ins Herz überzugehen begann, das heisst, das Herz fing an beim gewöhnlichen Schlagen, gleichsam innerlich, die Gebetsworte mit jedem Schlag auszusprechen. ${ }^{65}$ Das führt schliesslich dazu, dass auch seine bewusste Aufmerksamkeit beanspruchende Tätigkeiten keine Unterbrechung des Gebets mehr zur Folge haben ${ }^{66}$ und er sogar im Schlaf weiterbetet. ${ }^{67}$ Die weiteren Schritte und Erlebnisse des Pilgers seien hier nur angedeutet als Momente innigsten Ergriffenseins, die stets einen Bezug auf seine konkrete Umgebung haben und deutlich in Bezug mit seinen sozialen Beziehungen stehen. ${ }^{68}$ Der Pilger hat erreicht, was ihn seine Reise hat antreten lassen: zu verstehen, was das immerwährende Gebet ist und es selbst ausüben zu können.

Einstellung der Sorglosigkeit und Wachsamkeit.Vgl. Jungclaussen, Aufrichtige Erzählungen, $10 \mathrm{f}$.

${ }^{61}$ Vgl. Jill Gather, Teachings on the Prayer of the Heart in the Greek and Syrian Fathers. The Significance of Body and Community, Piscataway/NJ 2010,14, die auf die Drei-Stufen-Lehre bei Evagrius Ponticus rekurriert.

${ }^{62}$ Zur differenzierten Rolle, die der Leib hierbei spielt, vgl. Gather, Teachings, $17 f$.

${ }^{63}$ Paradigmatisch hierfür steht der Pilger, der sich nach dem Tod seiner Frau auf Wanderschaft begeben hatte, dabei in einem Gottesdienst auf 1 Thess 5,17 gestossen war und dann weiterpilgerte in dem Wunsch, das "Beten ohne Unterlass « zu verstehen und zu erlernen.

${ }^{64}$ Jungclaussen, Aufrichtige Erzählungen, 33-37.

${ }^{65}$ Ebd., 40.

${ }^{66}$ Wie z.B. das Zuhören: Jungclaussen, Aufrichtige Erzählungen, 48.

${ }^{67}$ Ebd., 60. Schliesslich kommt bei ihm auch noch die Verbindung mit dem Atmen hinzu, ebd., 58.

${ }^{68}$ Ebd., 37, 50, 67. Der Pilger setzt seine unmittelbaren Erfahrungen immer ins Verhältnis mit dem Gebet; in einer Kirche, in der er ein paar Monate arbeitet, beginnen die Menschen seine Nähe zu suchen. 


\section{b. Zeit und Gebet}

Es geht beim immerwährenden Gebet also um eine Praxis leiblichen Vollzugs des Gebets, das mittels der unaufhörlichen Wiederholung die bewusst vollzogene Aussprache unterwandert und über den Atem hinein "in Fleisch und Blut« übergeht. Das hat zur Folge, dass das Gebet ein Eigenleben entfaltet und die Beterin auch in ihrer präbewussten Disposition ausrichtet. Es kommt, wie es bei Luther mit dem Predigen des Geistes angeklungen ist, zu einem Subjektwechsel des Gebets: nicht mehr ich bete, sondern es betet in mir und setzt mir jene präsent, die neben mir immer schon da waren. ${ }^{69}$ Insofern verbindet sich hier das Moment des rhythmisierten, den Wechseln der Zeit ausgesetzten Gebets, das insofern auch als ein eigentliches Werk bestehen bleibt, mit dem Moment des fortlaufenden Bewusstseins eines Gottgedenkens, dem nicht mehr nur die Worte der Bibel, sondern schlechthin alles zum "Feuerzeug" wird. Das Herzensgebet stellt, indem es an eben jenem Konnex von Zeit und Selbst angesiedelt und dauerhaft selbsttätig wird, eine Figur des kontinuierlichen Zusammenfalls von Zeitentzug und Zeitgewährung dar und damit von Ewigkeit in der Zeit.

- Dipl. theol. Johanna Breidenbach ist Assistentin am Lehrstuhl für Systematische Theologie an der Theologischen Fakultät der Universität Zürich.

${ }^{69}$ Auf die Verschränkung von Herzensgebet und Orientierung am Nächsten macht besonders aufmerksam Nastja Makarytschowa, Mitte des Herzens, Zürich/Paderborn/Wien 1957, 21-26; 43-46. 